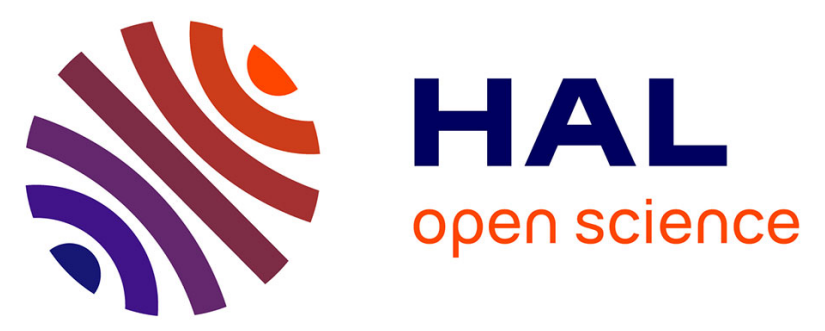

\title{
Temperature sensors based on europium polyoxometalate and mesoporous terbium metal-organic framework
}

Cédric Viravaux, Olivier Oms, Anne Dolbecq, Emma Nassar, Lucas Busson, Caroline Mellot-Draznieks, Rémi Dessapt, Hélène Serier-Brault, Pierre Mialane

\section{To cite this version:}

Cédric Viravaux, Olivier Oms, Anne Dolbecq, Emma Nassar, Lucas Busson, et al.. Temperature sensors based on europium polyoxometalate and mesoporous terbium metal-organic framework. Journal of Materials Chemistry C, 2021, 10.1039/d1tc01532j . hal-03277059

\section{HAL Id: hal-03277059 \\ https://hal.science/hal-03277059}

Submitted on 5 Jul 2021

HAL is a multi-disciplinary open access archive for the deposit and dissemination of scientific research documents, whether they are published or not. The documents may come from teaching and research institutions in France or abroad, or from public or private research centers.
L'archive ouverte pluridisciplinaire HAL, est destinée au dépôt et à la diffusion de documents scientifiques de niveau recherche, publiés ou non, émanant des établissements d'enseignement et de recherche français ou étrangers, des laboratoires publics ou privés. 


\title{
ARTICLE
}

\section{Temperature sensors based on europium polyoxometalate and mesoporous terbium metal-organic framework}

Received 00th January 20xx, Accepted 00th January 20xx DOI: $10.1039 / x 0 x \times 00000 x$

\author{
Cédric Viravaux, ${ }^{a}$ Olivier Oms, ${ }^{\mathrm{a}}$ Anne Dolbecq, ${ }^{\mathrm{a}}$ Emma Nassar, ${ }^{\mathrm{a}}$ Lucas Busson, ${ }^{\mathrm{a}}$ Caroline Mellot- \\ Draznieks, ${ }^{b}$ Rémi Dessapt, ${ }^{c}$ Hélène Serier-Brault ${ }^{{ }^{*}}$ and Pierre Mialane ${ }^{\mathrm{a}^{*}}$
}

\begin{abstract}
The first example of ratiometric luminescent thermometers based on lanthanide complexes encapsulated in a lanthanide metal-organic framework (MOF) is reported. In EuW ${ }_{10} @ \mathrm{~Tb}$-TATB, the $\left[\mathrm{EuW}_{10} \mathrm{O}_{36}\right]^{9-}$ polyoxometalate (POM) is incorporated into the cavities of the mesoporous terbium MOF Tb-TATB with two different POM loadings (9.1 and $19.5 \mathrm{wt} \%)$. Eu ${ }^{3+}$ and $\mathrm{Tb}^{3+}$ ions of the POM and MOF, respectively, act as dual emitters. Optical measurements evidence efficient $\mathrm{Tb}^{3+}$-to-Eu ${ }^{3+}$ energy transfer, suggesting that the EuW $\mathrm{W}_{10}$ units are close to terbium centers. These observations are supported by computational investigations whereby a favored localisation of the EuW $\mathrm{W}_{10} \mathrm{POM}$ in the MOF's pores is found close to Tbcenters evidencing hydrogen bond type interactions between terminal oxygen atoms of the POM and Tb-coordinated water molecules. The reported materials act as temperature sensors in the physiological domain, exhibiting high relative thermal sensitivities of $2.68 \% \mathrm{~K}^{-1}$ and $2.37 \% \mathrm{~K}^{-1}$ at $300 \mathrm{~K}$ for the $9.1 \mathrm{wt} \%$ and $19.5 \mathrm{wt} \%$ composites, respectively, with a thermal uncertainty of $0.09 \mathrm{~K}$.
\end{abstract}

\section{Introduction}

The field of luminescent thermometers is in expansion due to the numerous advantages of non-contact devices which can find applications in domains ranging from biomedicine to electronics. ${ }^{1-3}$ Best thermometric performances are obtained with ratiometric sensors where two discriminable emission peaks are used, preventing effects of optoelectronic drifts of excitation source or detectors. Many reported ratiometric temperature sensors are based on various lanthanide materials such as fluorides 4,5 or, more recently, coordination polymers and metal-organic frameworks. ${ }^{6}$ The latter indeed represent excellent platforms for engineering light-emitting centers and tuning photoluminescence properties. Not surprisingly, lanthanide-bearing MOFs (denoted as LnMOFs) have been the most studied ones. ${ }^{6}$ The first LnMOFs ratiometric thermometer, based on two emissions of $\mathrm{Tb}^{3+}$ at $545 \mathrm{~nm}$ and $\mathrm{Eu}^{3+}$ at $613 \mathrm{~nm}$, have been reported by Cui et al. in 2012. ${ }^{7}$ Since then, several Eu/Tb mixed LnMOFs have been prepared, with maximal sensitivities reached at temperature ranging from cryogenic to $450 \mathrm{~K} .{ }^{8-15}$

Also, the porosity of LnMOFs with a single emitting $\mathrm{Ln}^{3+}$ ion

\footnotetext{
a. Université Paris-Saclay, UVSQ, CNRS, Institut Lavoisier de Versailles, 78000 Versailles, France.E-mail : pierre.mialane@uvsq.fr

b. Laboratoire de Chimie des Processus Biologiques, UMR CNRS 8229, Collège de France, Sorbonne Université, PSL Research University, 11 Place Marcelin Berthelot, 75231 Paris Cedex 05, France.

c. Université de Nantes, CNRS, Institut des Matériaux Jean Rouxel, IMN, F-44000 Nantes, France.E-mail : helene.brault@cnrs-imn.fr

tElectronic Supplementary Information (ESI) available: Detailed synthesis and characterizations. See DOI: 10.1039/x0xx00000x
}

can be used to design ratiometric thermometer where the guest molecule acts as a second emitter. However, the incorporation of organic dyes into porous LnMOFs has been scarcely reported so far. A dual-emitting perylene@EuMOF acting as ratiometric temperature sensor in the physiological temperature range has been synthesized via an impregnation process using the ZJU-88 MOF $\left(\mathrm{S}_{\mathrm{r}}=1.28 \%\right.$ at $\left.293 \mathrm{~K}\right) .{ }^{16} \mathrm{In}$ addition, a coumarin derivative $\mathrm{C} 460$ has been encapsulated in a TbMOF, exhibiting a high thermal sensitivity of $4.48 \%$ at 300 $\mathrm{K},{ }^{17}$ while very recently Coumarin 7 was immobilized in a trimesate EuMOF with moderate thermometric properties $\left(\mathrm{S}_{\mathrm{r}}\right.$ $=0.5 \%$ at $363 \mathrm{~K}) .{ }^{18}$ Nevertheless, to the best of our knowledge, the incorporation of lanthanide complexes into LnMOFs for thermometry remains unexplored. Polyoxometalates (POMs) incorporating lanthanides ${ }^{19,20}$ appear as ideal candidates, as they combine excellent luminescence properties (narrow emission bands, large Stokes shift and long lifetime), high stability and rigidity. Furthermore, the incorporation of POMs into MOFs has been extensively studied, in particular for catalytic purposes. ${ }^{21}$ It is worth mentioning that the nonluminescent $\mathrm{Ln}$-free POM [ $\left.\mathrm{SiMo}_{12} \mathrm{O}_{40}\right]^{4-}$ has been also used for templating the formation of Eu/Tb mixed LnMOF for temperature sensing purposes. ${ }^{22}$ In addition, we recently reported the incorporation of the highly luminescent europium-containing POM $\left[\mathrm{EuW}_{10} \mathrm{O}_{36}\right]^{9-}\left(\mathrm{EuW}_{10}\right)$ into the UiO$67 \mathrm{MOF}$, where $\mathrm{Eu}^{3+}$ ions and the organic linker of the framework act as dual emitters. However, its relative thermal sensitivity was quite low (up to $0.26 \% \mathrm{~K}^{-1}$ at $303 \mathrm{~K}$ ) as a result of the very limited luminescent performances of the organic emitter. ${ }^{23}$

Herein, we report the encapsulation of the $\mathrm{EuW}_{10} \mathrm{POM}$ into the Tb-TATB MOF built of terbium tetranuclear units 
connected by TATB ligands $\left(\mathrm{H}_{3}\right.$ TATB $=$ triazine-1,3,5-tribenzoic acid) (Fig. 1a). ${ }^{24}$ This mesoporous MOF contains large nanoscopic distorted spherical cages of 3.9 and $4.7 \mathrm{~nm}$ diameters accessible through pentagonal and hexagonal windows of 1.3 and $1.7 \mathrm{~nm}$ aperture, respectively. This MOF has already been used as host for enzymes via impregnation in water ${ }^{25}$ and is well-suited for the incorporation of EuW 10 , whose dimensions are $0.9 \times 1.7 \mathrm{~nm}$. It is demonstrated here that this first dual-luminescent Ln complex@LnMOF composite behaves as a highly sensitive luminescent thermometer in the physiological domain.

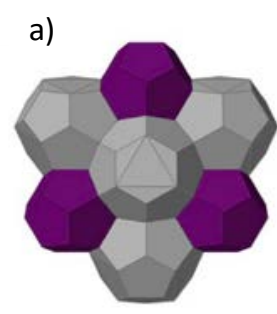

c)
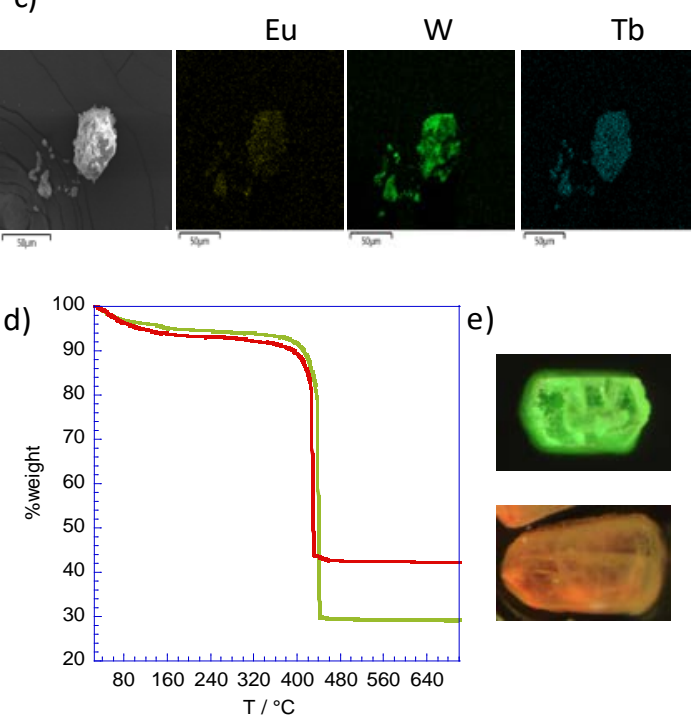

Fig. 1 a) left: truncated supertetrahedra forming the Tb-TATB MOF (from ref 26); right: the $\left[\mathrm{EuW}_{10} \mathrm{O}_{36}\right]^{9-}$ POM. Blue octahedra, $\mathrm{WO}_{6}$, pink sphere, Eu; b) IR spectra

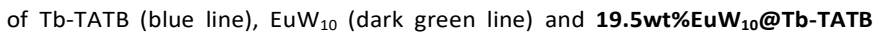
(red line). The vertical black line indicates the $\mathrm{W}=\mathrm{O}$ vibrations position; c) SEM image of 19.5wt\%EuW 10 $_{10}$ Tb-TATB and EDS elemental mapping of the Eu, W and Tb elements, respectively; d) TGA curves of Tb-TATB (green line) and 19.5wt\%EuW $10 @$ @Tb-TATB (red line); e) Images of Tb-TATB (top) and 19.5wt\%EuW 10 @Tb-TATB (bottom) under 365 nm irradiation.

\section{Results and discussion}

\section{Synthesis and characterizations of the composites}

$\left[\mathrm{Tb}_{16}(\mathrm{TATB})_{16}(\mathrm{DMA})_{24}\right] \cdot 91 \mathrm{DMA} \cdot 108 \mathrm{H}_{2} \mathrm{O}$ (DMA

dimethylacetamide) (Tb-TATB) has been prepared as previously described. ${ }^{24}$ The 19.5wt\%EuW $10 @$ @b-TATB composite was synthesized by mixing $\mathrm{Na}_{9}\left[\mathrm{EuW}_{10} \mathrm{O}_{36}\right]$ and $\mathrm{Tb}-$ TATB in aqueous medium, the resulting white material being thoroughly washed with water in order to remove any POM potentially deposited at the surface of the MOF particles. The infrared spectrum of 19.5wt\%EuW $\mathbf{W}_{10} @$ Tb-TATB mainly presents the feature of the MOF (Fig. S1, ESI $\dagger$ ). However, a band located at $920 \mathrm{~cm}^{-1}$ and attributed to $\mathrm{W}=\mathrm{O}$ vibrations (Fig. S1, ESI† and $1 \mathrm{~b}$ ) strongly suggests the presence of the POM. The latter is confirmed by SEM-EDS elemental mapping experiments, which show a uniform distribution of Eu, $\mathrm{W}$ and Tb elements throughout the material (Fig. 1C). In addition, the Tb:W:Eu ratio of 16:9:1.2 determined by EDS measurements indicates a ratio of 0.9:1 POM: $\left\{\mathrm{Tb}_{16}\right\}$ unit while no significant amount of sodium counterions was detected. This implies, as usually observed for POM@MOF materials, ${ }^{27}$ that the negative charges introduced by the POMs may be compensated by linker deficiencies. Thermogravimetric analysis (TGA) measurements show mass losses at $430^{\circ} \mathrm{C}$ of $70.5 \%$ for TbTATB and 57.5\% for 19.5wt\%EuW 10 @Tb-TATB (Fig. 1d). These weight losses are assigned to water removal in a first step and then to the linker's decomposition along with the formation of inorganic oxides. The lower weight loss of 19.5wt\%EuW $10 @$ @bTATB with respect to the pristine Tb-TATB solid is indeed in line with the presence of POMs in the POM@MOF material. The following chemical formula $\left[\mathrm{Tb}_{16}(\mathrm{TATB})_{13.3}\left(\mathrm{H}_{2} \mathrm{O}\right)_{24}\right]\left(\mathrm{EuW}_{10} \mathrm{O}_{36}\right)_{0.9^{\circ}}\left(\mathrm{H}_{2} \mathrm{O}\right)_{40}$ is thus proposed for 19.5wt\%EuW 10 @Tb-TATB, in accordance with both the total weight loss observed by TGA (exp. $57.5 \%$, calc. $56.3 \%$ ) and the water loss (exp. 9.7\%, calc. 8.2\%) and corresponding to a $\mathrm{Tb}_{0.95} \mathrm{Eu}_{0.05}$ molar composition. Besides, we can note that while the nature of the obtained large single-crystals of the TbTATB precursor has been checked by single-crystal X-Ray diffraction, affording the expected cell parameters, ${ }^{24}$ only low resolution powder X-Ray diffraction patterns were obtained for both the MOF and POM@MOF compounds (Fig. S2, ESI†). Finally, Fig. 1e and S3, ESI† show pictures of Tb-TATB and 19.5wt\%EuW10@Tb-TATB crystals under visible and UV-light irradiation. It can be clearly seen that the green luminescence of Tb-TATB turns orange after EuW $\mathrm{W}_{10}$ impregnation. With the aim to assess the influence of the POM loading on the luminescent performances, 9.1wt\%EuW $10 @$ Tb-TATB, corresponding to the $\left[\mathrm{Tb}_{16}(\mathrm{TATB})_{14.8}\left(\mathrm{H}_{2} \mathrm{O}\right)_{24}\right]\left(\mathrm{EuW}_{10} \mathrm{O}_{36}\right)_{0.4^{\circ}}\left(\mathrm{H}_{2} \mathrm{O}\right)_{40}$ composite was also prepared following a similar synthetic procedure but working at a lower POM:Tb-TATB ratio (see experimental section in the ESI for details).

\section{Computational investigations}

We further probed the likely positions of the EuW 10 POMs within the porous Tb-TATB through a computational approach using simulated annealing (SA) calculations (see ESI for details) as in previously reported procedures. ${ }^{28}$ Typically, the POMs are found located in the vicinity of Tb sites accessible at the MOF's internal surface, whereby they are stabilized by hydrogen bond interactions with water molecules coordinated to the Tb centers. Fig. 2 illustrates one such position of the POM as extracted from the lowest energy ones computed in SA calculations. The EuW 10 POM is shown here to benefit from multiple $\mathrm{H}$-bond interactions with two neighboring Tb centers, with $\mathrm{W}=\mathrm{O} \cdots \mathrm{H}\left(\mathrm{H}_{2} \mathrm{O}\right)$ distances in the 2.2-2.8 $\AA$ range, allowing here a $\mathrm{Tb} \cdots$ Eu distance of $6.3 \AA$. Interestingly, the simulations 
show that the EuW 10 POMs tend to locate preferably in the vicinity of $\mathrm{Tb}$ centers possessing water molecules in their coordination sphere, leaving $\mathrm{Tb}$ centers coordinated exclusively to TATB linkers unvisited.

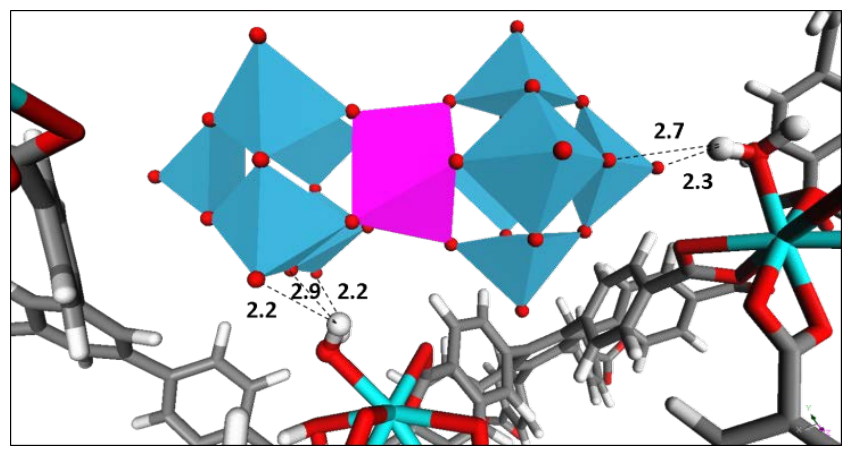

Fig. 2 Representation of EuW $10 @ T b-T A T B$ where the position of the POM is obtained from computations (see text). $\mathrm{WO}_{6}$, blue polyhedra; $\mathrm{EuO}_{8}$, pink polyhedron; $\mathrm{O}$, red spheres; $\mathrm{C}$, gray sticks; $\mathrm{Tb}$, turquoise sticks; $\mathrm{H}$, white sticks.

\section{Optical properties}

The photophysical properties of both 19.5wt\%EuW $10 @$ @bTATB and 9.1wt\%EuW $10 @$ @Tb-TATB were thoroughly investigated at room temperature. First, their emission spectra (Fig. S4, ESI†) display the characteristic green and red luminescence of $\mathrm{Tb}^{3+}$ and $\mathrm{Eu}^{3+}$ ions, respectively, with the typical lines at 490, 545, 581, and $619 \mathrm{~nm}$ attributed to the $\mathrm{Tb}^{3+}{ }^{5} \mathrm{D}_{4} \rightarrow{ }^{7} \mathrm{~F}_{6-3}$ transitions, and at 587, 611,652, and $695 \mathrm{~nm}$ for the $\mathrm{Eu}^{3+}{ }^{5} \mathrm{D}_{0} \rightarrow{ }^{7} \mathrm{~F}_{1-4}$ transitions. As already observed for EuW $_{10} @ U i O-67,{ }^{23}$ the intensity of the ${ }^{5} D_{0} \rightarrow{ }^{7} F_{2}$ transition with respect to that of the ${ }^{5} D_{0} \rightarrow{ }^{7} F_{1}$ magnetic dipole transition is significantly increased in the EuW $10 @ T b-T A T B$ composites when compared to the same transitions observed for the sodium salt of EuW $\mathrm{W}_{10}$, which confirms that the site symmetry of the $\mathrm{Eu}^{3+}$ ion in $\mathrm{EuW}_{10}$ decreases when the POM is introduced into the MOF pores.

The photoluminescence excitation (PLE) spectrum of 19.5wt\%EuW10@Tb-TATB monitored within the ${ }^{5} \mathrm{D}_{4} \rightarrow{ }^{7} \mathrm{~F}_{5} \mathrm{~Tb}^{3+}$ transition (Fig. S5, ESI†) displays mainly a broad band between 300 and $370 \mathrm{~nm}$, which is due to the $\pi-\pi^{*}$ transition of the TATB ligand, the $f-f$ lines being very weak. When monitored within the ${ }^{5} \mathrm{D}_{0} \rightarrow{ }^{7} \mathrm{~F}_{2} \mathrm{Eu}^{3+}$ transition, the PLE spectrum is composed of a broad band centered at $280 \mathrm{~nm}$, corresponding to the $\mathrm{O} \rightarrow \mathrm{W}$ ligand-to-metal charge transfer (LMCT) in the EuW $_{10}$ moiety, and of the MOF's ligand broad band together with the $\mathrm{f}-\mathrm{f}$ lines of $\mathrm{Eu}^{3+}$. Thus, the presence of the LMCT band confirms
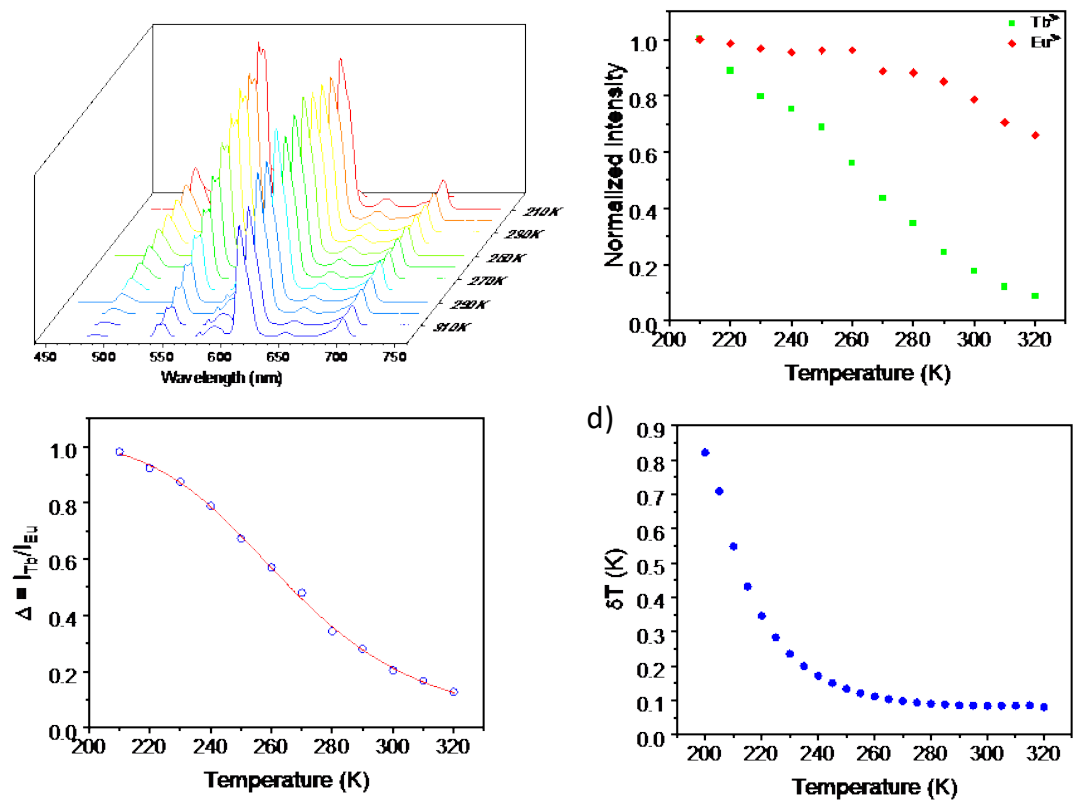

Fig. 3 a) Emission spectra of $9.1 \mathbf{w t} \% \mathrm{EW}_{\mathbf{1 0}} @$ Th-TATB in the 200-320 K range with the excitation fixed at $330 \mathrm{~nm}$; b) Corresponding temperature dependence of the $\mathrm{I}_{\mathrm{Tb}}$ (green) and $\mathrm{I}_{\mathrm{Eu}}(\mathrm{red})$ parameters; $\mathrm{c}$ ) Corresponding temperature dependence of $\Delta$ in the $200-320 \mathrm{~K}$ range and Mott-Seitz fit (red line, $\left.\mathrm{R}^{2}=0.999\right)$; $\mathrm{d}$ ) Corresponding temperature uncertainty $(\delta \mathrm{T}$ $\left.=1 / \mathrm{S}_{\mathrm{r}} \times \delta \Delta / \Delta\right) . \delta \Delta / \Delta$ was estimated by the average relative errors in the same temperature range $(0.2 \%)$.

the integrity of the POM EuW 10 in the POM@MOF composite, while the presence of the excitation bands of the MOF evidences an intermolecular MOF-to-Eu ${ }^{3+}$ energy transfer Hereafter, the excitation wavelength will be fixed at $330 \mathrm{~nm}$, which corresponds to the intersection between the PLE spectra monitored at $541 \mathrm{~nm}\left(\mathrm{~Tb}^{3+}\right.$ transition) and $617 \mathrm{~nm}$
( $\mathrm{Eu}^{3+}$ transition), where both emitters are equally sensitized. Moreover, a $\mathrm{Tb}^{3+}$-to-Eu ${ }^{3+}$ energy transfer is discerned at roomtemperature, as shown by the presence of the ${ }^{7} \mathrm{~F}_{6} \rightarrow{ }^{5} \mathrm{D}_{4}$ (at $483 \mathrm{~nm}) \mathrm{Tb}^{3+}$ transition in the excitation spectrum monitored within the ${ }^{5} \mathrm{D}_{0} \rightarrow{ }^{7} \mathrm{~F}_{2} \mathrm{Eu}^{3+}$ transition (Fig. S5 insert, ESI $\dagger$ ). Emission lifetimes were determined at room-temperature for 
both EuW10@Tb-TATB compounds and for Tb-TATB. For all samples, a monoexponential behavior was observed for the ${ }^{5} \mathrm{D}_{4}$ decay curve monitored at $541 \mathrm{~nm}$ ( $\mathrm{Tb}^{3+}$ transition) and for the ${ }^{5} \mathrm{D}_{0}$ decay curve monitored at $617 \mathrm{~nm}$ (Eu ${ }^{3+}$ transition), the different lifetimes being given in Table S1. Thus, the $\mathrm{Tb}^{3+}$-to$\mathrm{Eu}^{3+}$ energy transfer efficiency was determined from the equation (1):

$\eta_{E T}=1-\frac{\tau}{\tau_{0}}$

with $\tau$ and $\tau_{0}$ corresponding to the lifetimes of the donor in the presence and absence of the acceptor, respectively. Thus, the $\mathrm{Tb}^{3+}$-to-Eu ${ }^{3+}$ energy transfer amounts to $40 \%$ and $37 \%$ for 9.1wt\%EuW $10 @$ @Tb-TATB and 19.5wt\%EuW $10 @$ @Tb-TATB, respectively. Finally, as expected by computational investigations one may assume that the $\mathrm{EuW}_{10}$ entities are close enough to the $\mathrm{Tb}^{3+}$ ions of the MOF to lead to a nonnegligible $\mathrm{Tb}^{3+}$-to-Eu ${ }^{3+}$ energy transfer. This observation is indeed corroborated by the above SA calculations which point towards the vicinity of Tb centers in the POM's environment.

The application of the EuW $10 @$ Tb-TATB composites as ratiometric luminescent thermometers was characterized using the emission spectra in the 200-320 $\mathrm{K}$ range. As an illustrative example, Fig. 3a presents the temperature dependent emission spectra of 9.1wt\%EuW $\mathbf{W}_{10} @ T$ Tb-TATB in the

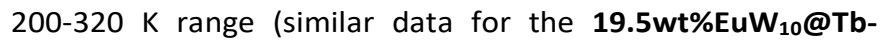
TATB material is reported in Fig. S6a, ESI†). Five consecutive emission spectra were recorded at each temperature. The temperature dependence of the $\mathrm{I}_{\mathrm{Tb}}$ (green) and $\mathrm{I}_{\mathrm{Eu}}$ (red) parameters is represented in Fig. 3b. The integrated areas of the ${ }^{5} D_{4} \rightarrow{ }^{7} F_{5}\left(I_{T b}\right)$ and of the ${ }^{5} D_{0} \rightarrow{ }^{7} F_{2}\left(I_{E U}\right)$ emissions were used to define the thermometric parameter as $\Delta=I_{T b} / I_{E u}, I_{T b}$ and $I_{E u}$ being obtained using the emission spectra in the $530-560 \mathrm{~nm}$ and 602-633 $\mathrm{nm}$ intervals, respectively.

The thermometric parameter $\Delta$ of both samples (Fig. $3 c$ and Fig. S6c, ESI $\dagger$ ) is depicted according to the temperature in the 200-320 K range. It may be described by the phenomenological Mott-Seitz expression involving a single deactivation channel assuming a temperature dependence of the $\mathrm{Eu}^{3+}$ emission smaller than that of the $\mathrm{Tb}^{3+}$ emission (Fig. $3 \mathrm{~b}$ and Fig. S6b, $\mathrm{ESI} \dagger$ ) according to the equation (2):

$\Delta(T)=\frac{\Delta_{0}}{1+\alpha \exp \left(-\Delta E / k_{B} T\right)}$

where $\Delta_{0}$ is the $\Delta$ parameter at $\mathrm{T} \rightarrow 0 \mathrm{~K}, \alpha=\mathrm{W}_{0} / \mathrm{W}_{\mathrm{R}}$ is the ratio between the non-radiative and radiative decay rates in the limit of $\mathrm{T} \rightarrow 0 \mathrm{~K}$ and $\Delta \mathrm{E}$ is the energy difference between the emitting level $\left({ }^{5} D_{4}\right)$ and the level responsible for its nonradiative deactivation. Fitting Equation (2) to the experimental $\Delta(T)$ curves, we find excellent correlation coefficients attesting the adequacy of this simple model to get the calibration curve of the hybrid materials (Fig. 3c and Fig. $\mathrm{S} 6 \mathrm{c}, \mathrm{ESI} \dagger)$. Finally, the $\Delta \mathrm{E}$ value was found equal to $2119 \pm 70$ $\mathrm{cm}^{-1}$ for 9.1wt\%EuW 10 @Tb-TATB and $1932 \pm 65 \mathrm{~cm}^{-1}$ for 19.5wt\%EuW10@Tb-TATB, which can be ascribed to the backenergy transfer from the ${ }^{5} \mathrm{D}_{4}$ level of $\mathrm{Tb}^{3+}\left(20500 \mathrm{~cm}^{-1}\right)^{6}$ to the triplet level of the TATB ligand $\left(T_{1}=21380 \mathrm{~cm}^{-1}\right) .^{29}$ This is confirmed by the thermal evolution of ${ }^{5} D_{4}$ lifetimes which decreases when the temperature increases whereas the ${ }^{5} D_{0}$ lifetime is almost constant (Fig. S7 and S8, ESI†). The same ${ }^{5} \mathrm{D}_{4}$ lifetime thermal quenching was also observed on the Tb-TATB MOF (Fig. S9, ESI†), indicating that the EuW 10 entity plays the role of reference in the ratiometric thermometers. Then, in order to evaluate the thermal performances of mixed compounds, we calculated the relative thermal sensitivity defined as $S_{r=}|\partial \Delta / \partial T| / \Delta$ which is a usual figure of merit to compare the performance of distinct systems. ${ }^{30}$ Both materials exhibit good thermal sensitivity in the physiological range (293-323 K) with a maximum of relative thermal sensitivity of $2.68 \% \mathrm{~K}^{-1}$ at $300 \mathrm{~K}$ for $9.1 \mathrm{wt} \% \mathrm{EuW}_{10} @ \mathrm{~Tb}-\mathrm{TATB}$, and $2.37 \mathrm{KK}^{-1}$ for 19.5wt\%EuW 10 @Tb-TATB (Fig. 4). These values are higher than those of most previously reported luminescent thermometers operating in the physiological range. Finally, the temperature uncertainty $\delta T=1 / S_{r} \times \delta \Delta / \Delta$, which defines the smallest temperature change that may be ascertained in a given measurement, was evaluated as amounting to $0.08 \mathrm{~K}$ for both samples on a wide temperature range (from 280 to 320 $\mathrm{K})$, which makes them very promising materials for biological applications (Fig. 3d and S6d, ESI†).

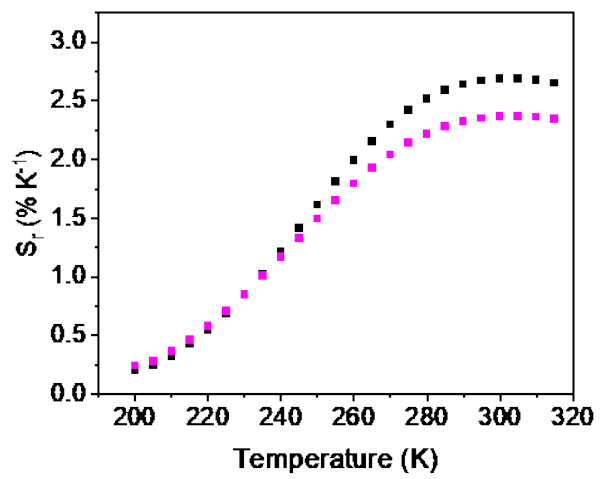

Fig. 4 Relative thermal sensitivity of 9.1wt\%EuW ${ }_{10} @$ Tb-TATB (black line) and 19.5wt\%EuW 10 @Tb-TATB (pink line) in the 200-320K range.

\section{Conclusions}

The red emitting EuW 10 POM was successfully incorporated into the mesoporous green emitting Tb-TATB MOF. According to the POM:MOF ratio used for the impregnation step, two composites,

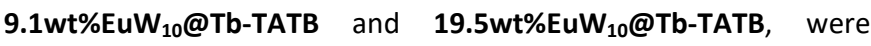
prepared and characterized. The temperature-dependence of their optical properties were investigated and undoubtedly pointed out an energy transfer from the Tb MOF network to the $\mathrm{Eu}^{3+}$ centers. The latter is supported by the computed positioning of the EuW 10 within the Tb-TATB host whereby it is favorably immobilized near Tb-centers through hydrogen bond type interactions with Tbcoordinated water molecules. As a result, these composites appear as effective ratiometric luminescent thermometers, their relative thermal sensitivity values $\left(2.68 \% \mathrm{~K}^{-1}\right.$ for 9.1wt\%EuW ${ }_{10} @ T$ Tb-TATB and $2.37 \% \mathrm{~K}^{-1}$ for $19.5 w_{t} \% \mathrm{EuW}_{10} @$ @Tb-TATB at $300 \mathrm{~K}$ ) clearly evidencing their high optical performance in the physiological 
domain. These composites constitute the first members of a new family of hybrid dual-emitting POM@MOF materials. The richness of the POMs and MOFs chemistry makes it possible to envisage a multitude of related systems, including other LnPOM@LnMOFs composites with tunable POM/MOF interactions, but also the coincorporation of POMs with other fluorophores (quantum dots,...) in non-luminescent MOFs for the development of new POM-based luminescent thermometers. Both strategies are currently under investigation in our groups.

\section{Experimental section}

All reagents were purchased and used without further purification except $\quad \mathrm{Na}_{9}\left[\mathrm{EuW}_{10} \mathrm{O}_{36}\right] \cdot 32 \mathrm{H}_{2} \mathrm{O} \quad\left(\mathrm{EuW}_{10}\right)^{31}$ and $\left[\mathrm{Tb}_{16}(\mathrm{TATB})_{16}(\mathrm{DMA})_{24}\right] \bullet 91 \mathrm{DMA} \bullet 108 \mathrm{H}_{2} \mathrm{O} \quad(\text { Tb-TATB })^{24}$ which were synthesized according to reported procedures. Prior to the impregnation of the polyoxometalate, Tb-TATB was washed with water and ethanol and dried overnight.

Preparation of $19.5 w t \%$ EuW $_{10} @ T$ Tb-TATB. In a scintillation vial, 24 $\mathrm{mg}$ of $\operatorname{EuW}_{10}(7.3 \mu \mathrm{mol})$ were dissolved in $3 \mathrm{~mL}$ of water. Then, 24 $\mathrm{mg}$ of crushed Tb-TATB $(1.1 \mu \mathrm{mol})$ was added, and the suspension stirred at $60^{\circ} \mathrm{C}$ for one night using a shaker. The obtained white powder was then filtrated, thoroughly washed with water, ethanol and dried overnight $(\mathrm{m}=18 \mathrm{mg})$. EDS: Tb : $\mathrm{W}$ : Eu calc. : $16: 9: 0.9$, exp. : $16: 9$ : 1.2. IR (v/cm-1): $1421(\mathrm{~m}), 1393$ (s), 1377 (s), 1353 (vs), $1136(\mathrm{~m}), 1103(\mathrm{~m}), 1015(\mathrm{~m}), 976(\mathrm{w}), 922(\mathrm{w}), 868(\mathrm{w}), 830(\mathrm{~m})$, $798(\mathrm{~m}), 771(\mathrm{~s}), 699(\mathrm{~m}), 543(\mathrm{~m}), 493(\mathrm{~s})$.

Preparation of 9.1wt\%EuW $10 @ T$ Tb-TATB. This compound was prepared as described for 19.5wt\%EuW $10 @$ @Tb-TATB but using 16 $\mathrm{mg}$ of Tb-TATB $(0.7 \mu \mathrm{mol})$ and $3 \mathrm{mg}$ of $\operatorname{EuW}_{10}(0.9 \mu \mathrm{mol}) .13 \mathrm{mg}$ of 9.1wt\%EuW10@Tb-TATB were finally obtained. EDS: Tb : W calc. : 16 : 4, exp.: 16 : 4. IR (v/cm $\left.{ }^{-1}\right): 1423(\mathrm{~m}), 1399$ (s), 1378 (s), 1359 (vs), $1137(\mathrm{~m}), 1103(\mathrm{~m}), 1015(\mathrm{~m}), 931(\mathrm{w}), 868(\mathrm{w}), 829(\mathrm{~m}), 796$ (m), $774(\mathrm{~s}), 702(\mathrm{~m}), 552(\mathrm{~m}), 496(\mathrm{~s})$.

\section{Author Contributions}

The project was conceived and designed by PM with significant input from $A D$ and $O O . C V, E N$ and $L B$ prepared the samples and performed the characterizations. $C M D$ carried out the computational investigations. RD and HSB performed the optical measurements. The first manuscript draft was prepared by PM, HSB and CMD. All the authors reviewed the manuscript and participated actively in the discussion of the results.

\section{Acknowledgements}

Y. Benseghir is gratefully acknowledged for EDS and EDS mapping analysis. K. Boukheddaden and G. Bouchez are warmly thanked for pictures of crystals. This work was supported by the Ministère de I'Enseignement Supérieur et de la Recherche, the CNRS, the Université de Versailles Saint Quentin en Yvelines, the Université de Nantes, the Collège de France and a public grant overseen by the French National Research Agency (ANR) as part of the

"Investissements d'Avenir" program n ${ }^{\circ}$ ANR-11-IDEX-0003-02 and CHARMMMAT ANR-11-LABX-0039.

\section{Conflicts of interest}

There are no conflicts to declare.

\section{References}

1

2

3

\section{4}

4

5

7

F. Wang, Y. Han and N. Gu, ACS Sensors, 2021, 6, 290-302. R. Li, F. F. Xu, Z. L. Gong and Y. W. Zhong, Inorg. Chem. Front., 2020, 7, 3258-3281.

A. Bednarkiewicz, L. Marciniak, L. D. Carlos and D. Jaque, Nanoscale, 2020, 12, 14405-14421.

F. Vetrone, R. Naccache, A. Zamarrón, A. J. De La Fuente, F. Sanz-Rodríguez, L. M. Maestro, E. M. Rodriguez, D. Jaque, J. G. Sole and J. A. Capobianco, ACS Nano, 2010, 4, 32543258.

A. Skripka, A. Benayas, R. Marin, P. Canton, E. Hemmer and F. Vetrone, Nanoscale, 2017, 9, 3079-3085.

J. Rocha, C. D. S. Brites and L. D. Carlos, Chem. - A Eur. J., 2016, 22, 14782-14795.

Y. Cui, H. Xu, Y. Yue, Z. Guo, J. Yu, Z. Chen, J. Gao, Y. Yang, G. Qian and B. Chen, J. Am. Chem. Soc., 2012, 134, 39793982.

Z. Wang, D. Ananias, A. Carné-Sánchez, C. D. S. Brites, I. Imaz, D. Maspoch, J. Rocha and L. D. Carlos, Adv. Funct. Mater., 2015, 25, 2824-2830.

D. Ananias, C. D. S. Brites, L. D. Carlos and J. Rocha, Eur. J. Inorg. Chem., 2016, 2016, 1967-1971.

I. N'Dala-Louika, D. Ananias, C. Latouche, R. Dessapt, L. D. Carlos and H. Serier-Brault, J. Mater. Chem. C, 2017, 5, 10933-10937.

X. Liu, S. Akerboom, M. De Jong, I. Mutikainen, S. Tanase, A. Meijerink and E. Bouwman, Inorg. Chem., 2015, 54, 11323-11329.

A. Cadiau, C. D. S. Brites, P. M. F. J. Costa, R. A. S. Ferreira, J. Rocha and L. D. Carlos, ACS Nano, 2013, 7, 7213-7218. D. Zhao, D. Yue, K. Jiang, L. Zhang, C. Li and G. Qian, Inorg. Chem., 2019, 58, 2637-2644.

L.-L. Wu, J. Zhao, H. Wang and J. Wang, CrystEngComm, 2016, 18, 4268-4271.

Y. Yang, L. Chen, F. Jiang, M. Yu, X. Wan, B. Zhang and M. Hong, J. Mater. Chem. C, 2017, 5, 1981-1989.

Y. Cui, R. Song, J. Yu, M. Liu, Z. Wang, C. Wu, Y. Yang, Z. Wang, B. Chen and G. Qian, Adv. Mater., 2015, 27, 14201425.

T. Xia, T. Song, Y. Cui, Y. Yang and G. Qian, Dalton Trans., 2016, 45, 18689-18695.

J. Liu, X. Yue, Z. Wang, X. Zhang and Y. Xu, J. Mater. Chem. C, 2020, 8, 13328-13335.

C. Boskovic, Acc. Chem. Res., 2017, 50, 2205-2214.

B. S. Bassil and U. Kortz, Zeitschrift für Anorg. und Allg.

Chemie, 2010, 636, 2222-2231.

P. Mialane, C. Mellot-Draznieks, P. Gairola, M. Duguet, Y. 
Benseghir, O. Oms and A. Dolbecq, Chem Soc Rev, DOI:10.1039/d0cs00323a.

22 A. M. Kaczmarek, J. Mater. Chem. C, 2018, 6, 5916-5925.

23 W. Salomon, A. Dolbecq, C. Roch-Marchal, G. Paille, R. Dessapt, P. Mialane and H. Serier-Brault, Front. Chem., 2018, 6, 425.

24 Y. K. Park, S. B. Choi, H. Kim, K. Kim, B.-H. Won, K. Choi, J.-S. Choi, W.-S. Ahn, N. Won, S. Kim, D. H. Jung, S.-H. Choi, G.H. Kim, S.-S. Cha, Y. H. Jhon, J. K. Yang and J. Kim, Angew. Chem. Int. Ed., 2007, 46, 8230-8233.

25 V. Lykourinou, Y. Chen, X. Sen Wang, L. Meng, T. Hoang, L. J. Ming, R. L. Musselman and S. Ma, J. Am. Chem. Soc., 2011, 133, 10382-10385.

26 R. J. Drout, L. Robison and O. K. Farha, Coord. Chem. Rev., 2019, 381, 151-160.

27 W. Salomon, C. Roch-Marchal, P. Mialane, P. Rouschmeyer, C. Serre, M. Haouas, F. Taulelle, S. Yang, L. Ruhlmann and A. Dolbecq, Chem. Commun., 2015, 51, 2972-2975.

28 Y. Benseghir, A. Lemarchand, M. Duguet, P. Mialane, M. Gomez-Mingot, C. Roch-Marchal, T. Pino, M. H. Ha-Thi, M. Haouas, M. Fontecave, A. Dolbecq, C. Sassoye and C. Mellot-Draznieks, J. Am. Chem. Soc., 2020, 142, 94289438.

29 X. Zeng, J. Hu, M. Zhang, F. Wang, L. Wu and X. Hou, Anal. Chem., 2020, 92, 2097-2102.

30 C. D. S. Brites, A. Millán and L. D. Carlos, in Handbook on the Physics and Chemistry of Rare Earths, 2016, vol. 49.

31 M. Sugeta and T. Yamase, Bull. Chem. Soc. Jpn., 1993, 66, 444-449. 